\title{
Large Eddy Simulation of Thermally Induced Oscillations in the Convective Boundary Layer
}

\author{
Marcus Oliver Letzel And Siegfried RaAsch \\ Institute of Meteorology and Climatology, University of Hannover, Hanover, Germany
}

(Manuscript received 2 August 2002, in final form 8 April 2003)

\begin{abstract}
Mesoscale circulations induced by differential boundary layer heating due to surface inhomogeneities on scales of $5 \mathrm{~km}$ and more can significantly change the average properties and the structure of the convective boundary layer (CBL) as well as trigger off temporal oscillations. The results of one of the first numerical case studies using large eddy simulation (LES) on the mesoscale suggest that mesoscale circulations exhibit a considerably larger average kinetic energy than convection under homogeneous conditions. This affects turbulent transport processes and should be accounted for in larger-scale models even if their turbulence parameterizations rely on homogeneous control runs of high-resolution models.

This case study uses the Hannover parallelized large eddy simulation model (PALM) with prescribed 1D sinusoidal surface heat flux variations on wavelengths from 2.5 to $40 \mathrm{~km}$. The resulting mesoscale circulations are analyzed by means of domain-averaged cross sections, time averaged and normalized with the boundary layer height, as well as power spectra and domain-averaged time series.

The simulated mesoscale circulations were periodic. Vertical profiles and time series demonstrate that the onset of the mesoscale circulation triggers off a temporal boundary layer oscillation, whose period and amplitude depend on the surface heat flux perturbation wavelength and amplitude and on the background wind component perpendicular to the surface inhomogeneity orientation. Spectral analysis shows that the mesoscale circulations damp convection equally in all directions. A hypothesis of the oscillation mechanism is briefly discussed.
\end{abstract}

\section{Introduction}

Inhomogeneity on a wide range of spatial and temporal scales is a key feature of the earth's surface. Variability in, for example, terrain, vegetation, soil texture and wetness, cloud cover, and land use leaves its first imprints on the atmosphere in the atmospheric boundary layer (ABL). The sudden or gradual changes in radiative, thermal, moisture, and aerodynamic surface properties such as surface heat, momentum and humidity fluxes, surface roughness, temperature, wetness, and elevation affect the ABL flow structure and associated atmospheric processes (e.g., alter convection or precipitation).

This paper is concerned with the thermal effects that surface heterogeneities can exert on the ABL. Via differential heating heterogeneous surface heat fluxes produce horizontal pressure gradients that can initiate a mesoscale circulation. The effect of such a thermally induced mesoscale circulation (TMC) can extend well into the free atmosphere up to the midtroposphere (Dalu et al. 2000).

Corresponding author address: Marcus Letzel, Institute of Meteorology and Climatology, University of Hannover, Herrenhäuser Str. 2, 30419 Hanover, Germany.

E-mail: letzel@muk.uni-hannover.de
Heterogeneous surface information usually translates into the ABL dynamically (Belcher and Hunt 1998; Wood 2000) or thermally (Atkinson 1981; Segal and Arritt 1992). Both processes have been the subject of active research over the past two decades (cf. the reviews by Giorgi and Avissar 1997; Arnfield 2000; Mahrt 2000; Pielke 2001), with special emphasis on the parameterization of subgrid-scale effects of landscape heterogeneity on the resolved scales of mesoscale or global models.

Whereas most experimental investigations are focused on topography-related dynamical effects-for example, internal boundary layers due to flow over changing surface roughness-recent years have seen a growing amount of experimental evidence that thermal and/ or moisture surface heterogeneities can also have a strong impact on ABL flow structure (Mahrt et al. 1994; Weaver and Avissar 2001), though there is still an ongoing debate as to whether topographic or land use differences are more important (Shaw and Doran 2001).

Theoretical approaches primarily aim at improved parameterization schemes. Avissar and Chen (1993) introduced the new property "mesoscale kinetic energy" in order to represent the mesoscale fluxes in large-scale atmospheric models. An analytical study by Dalu and Pielke (1993) showed that the intensity of mesoscale 
cells induced by thermal surface heterogeneity is strongest for forcing wavelengths on the order of the local Rossby radius. Using similarity theory, Lynn et al. (1995, 2001), Zeng and Pielke (1995), and Arola (1999) proposed parameterization schemes for such mesoscale fluxes. Further theoretical approaches included, for example, a stochastic linear theory by Wang et al. (1996), conceptual statistical considerations by Avissar (1998), a thermodynamical theory by Souza et al. (2000) and the initial value problem studied by Dalu et al. (2000).

Numerical modeling of thermal effects of surface heterogeneities started more than $10 \mathrm{yr}$ ago, focusing on the development and/or validation of parameterization schemes (i.e., as the ones mentioned above), as well as on a better understanding of the mesoscale phenomena themselves. For the former, 2D (Avissar and Pielke 1989; Pielke et al. 1991; Zeng and Pielke 1995) and 3D mesoscale models (Wang et al. 1998; Liu et al. 1999; Weaver and Avissar 2001) tend to be used, while for the latter, direct numerical simulation and large eddy simulations (LES) are predominant.

Hechtel et al. (1990) and Hadfield et al. (1991, 1992) first used LES models to simulate TMCs in the convective boundary layer (CBL). Hechtel et al. (1990), investigating the land surface heterogeneity observed near Chickasha, Oklahoma, during the 1983 Boundary Layer Experiment, could not detect any significant impact on the CBL statistics. In a case study, Hadfield et al. (1991, 1992) showed that, under zero background wind conditions, a few high-amplitude, one-dimensional sinusoidal surface sensible heat flux variations drive a mean circulation, the intensity of which increases substantially with the wavelength of the heat flux perturbation, but drastically weakens with only light ambient wind.

Two-dimensional surface sensible heat flux inhomogeneities were first analyzed in LES case studies by Shen and Leclerc (1995), Cai (1999), and Raasch and Harbusch (2001). Shen and Leclerc (1995) showed that only surface heat flux inhomogeneities of horizontal extent equal to or larger than the $\mathrm{ABL}$ height $z_{i}$ influence the CBL structure significantly, particularly the higher statistical moments. Surface inhomogeneities of larger scales were found to produce more vigorous energy transport in the CBL and larger near-surface temperature variances than do those of smaller scales. Cai (1999) found that an urban surface idealized by a 2D mosaic of large and small surface heat flux patches significantly changes turbulence spectra. Raasch and Harbusch (2001) found the structure of the 2D TMC to be very sensitive to both wavelength and shape of the inhomogeneities, as well as to surface heat flux amplitude, wind speed and also wind direction. They showed that moderate background winds do not necessarily eliminate all impacts of surface heterogeneity; whether or not this is the case depends strongly on the wind direction relative to the heterogeneous structures.

Zhong and Doran (1998), using a mesoscale model, pointed out that idealized surface inhomogeneities such as those used in the above LES studies may seriously overestimate the strength of the TMC and its associated parameters, and Albertson et al. (2001) used heterogeneous 2D remote sensing data to trigger their LES study. Nevertheless, recent numerical case studies (Avissar and Schmidt 1998, hereafter AS98; Gopalakrishnan and Avissar 2000; Gopalakrishnan et al. 2000; Baidya Roy and Avissar 2000) concentrated on idealized $1 \mathrm{D}$ surface heterogeneities. They concluded that the CBL is most significantly affected by surface heat flux heterogeneities with a characteristic length scale of 5$10 \mathrm{~km}$ and more, and that this impact be nonlinearly dependent on the mean surface heat flux. Gopalakrishnan and Avissar (2000) and Gopalakrishnan et al. (2000) showed that the same length-scale criterion applies to the width of 1D sinusoidal hills that also generate TMCs.

However, the results of AS98 show that, in a CBL forced with inhomogeneities as large as $40 \mathrm{~km}$, (a) the potential temperature is not constant with height, and (b) the vertical sensible heat flux profile departs considerably from the linear variation with height, as would be typical of a homogeneous CBL. AS98's statement that only results from the final quasi-steady state of the boundary layer evolution were discussed is in some contrast to these observations, leaving it questionable whether the concept of a homogeneous, quasi-steady boundary layer still holds for such large inhomogeneities.

With these open questions of AS98 as its starting point, this numerical study reveals for the first time that under certain conditions the TMC onset induces a temporal oscillation of ABL flow, which significantly changes both average statistical properties and mean profiles. It identifies the parameters on which the oscillation depends and provides a hypothesis of the oscillation mechanism. This oscillation serves to explain the AS98 temperature and heat flux profiles.

In order to bring out the new oscillation phenomenon clearly, this study also idealizes surface heat flux heterogeneity as simple sine waves. Simulations of complex heterogeneous conditions are currently under way.

\section{Numerical experiment}

\section{a. Model description}

The parallelized large eddy simulation model (PALM) used for this study has been developed over the last years by the LES Group of the Institute of Meteorology and Climatology at the University of Hannover (Raasch and Etling 1998; Raasch and Schröter 2001). Up-to-date model documentation is available online (Raasch 2002).

In its dry mode used here PALM solves the NavierStokes equations in Boussinesq form, the first law of thermodynamics, and the equation for turbulent kinetic 
TABLE 1. Cases with zero background wind $\left(t_{s}\right.$ is simulation time, other parameters defined in the text).

\begin{tabular}{lccccc}
\hline \hline Case & $\begin{array}{c}D_{x} \\
(\mathrm{~km})\end{array}$ & $\begin{array}{c}\lambda_{x} \\
(\mathrm{~km})\end{array}$ & $\begin{array}{c}\overline{w^{\prime} \theta_{\mathrm{av}}^{\prime}} \\
\left(\mathrm{K} \mathrm{m} \mathrm{s}^{-1}\right)\end{array}$ & $\begin{array}{c}A_{x} \\
\left(\mathrm{~K} \mathrm{~m} \mathrm{~s}^{-1}\right)\end{array}$ & $\begin{array}{c}t_{s} \\
(\mathrm{~h})\end{array}$ \\
\hline A15 & 40 & 40 & 0.24 & 0.20 & 12 \\
A15h & 40 & - & 0.24 & 0 & 12 \\
B5 & 40 & 40 & 0.12 & 0.10 & 12 \\
B5h & 40 & - & 0.12 & 0 & 12 \\
L1 & 10 & 2.5 & 0.16 & 0.15 & 6 \\
L1h & 10 & - & 0.16 & 0 & 6 \\
L2 & 10 & 5.0 & 0.16 & 0.15 & 6 \\
L3 & 15 & 7.5 & 0.16 & 0.15 & 6 \\
L4 & 20 & 10 & 0.16 & 0.15 & 9 \\
L4A & 20 & 10 & 0.16 & 0.10 & 9 \\
L4B & 20 & 10 & 0.16 & 0.05 & 9 \\
L4C & 20 & 10 & 0.16 & 0.03 & 9 \\
L4D & 20 & 10 & 0.16 & 0.01 & 9 \\
L4h & 20 & - & 0.16 & 0 & 11 \\
L5 & 30 & 15 & 0.16 & 0.15 & 9 \\
L6 & 40 & 20 & 0.16 & 0.15 & 9 \\
L7 & 50 & 25 & 0.16 & 0.15 & 9 \\
L7h & 50 & - & 0.16 & 0 & 9 \\
L8 & 30 & 30 & 0.16 & 0.15 & 11 \\
\hline
\end{tabular}

energy (TKE). Nondivergent flow is assured by solving a Poisson equation for the so-called pressure perturbation $p$ using fast Fourier transforms (FFT). Subgridscale (SGS) turbulence is parameterized according to the suggestions of Deardorff (1980) with minor alterations. This study uses PALM's standard finite differences method for the discretisation of the differential equations and the leap-frog scheme for the time integration. Lateral boundary conditions are cyclic, and Monin-Obukhov similarity is assumed in the Prandtl layer between the surface and the first computational grid level. The roughness length $z_{0}$ is kept constant for all simulations $(0.1 \mathrm{~m})$.

\section{b. Experimental setup}

The starting point of this research was an open question of the AS98 study; the experimental design follows their case study to allow comparison.

The atmosphere was initialized with a weakly stable profile $\left(\partial \theta / \partial z=0.8 \mathrm{~K} \mathrm{~km}^{-1}\right.$, where $\theta$ is potential temperature and $z$ the vertical coordinate) up to a height of $1200 \mathrm{~m}$ and a strong capping inversion above $(\partial \theta / \partial z=$ $7.4 \mathrm{~K} \mathrm{~km}^{-1}$ ).

During the first $2 \mathrm{~h}$ after simulation start, a horizontally homogeneous near-surface vertical potential temperature flux $\overline{w^{\prime} \theta^{\prime}}$ av (hereafter, simply referred to as surface heat flux) was prescribed to let a quasi-steady boundary layer develop. Then, at $2 \mathrm{~h}$ after simulation start, one-dimensional sinusoidal variations of amplitude $A_{x}$ on wavelengths $\lambda_{x}$ from 2.5 to $40 \mathrm{~km}$ were imposed on the surface heat flux so that

$$
\overline{w^{\prime} \theta^{\prime}}(x)=\overline{w^{\prime} \theta^{\prime}}{ }_{\text {av }}+A_{x} \sin \left(\frac{2 \pi}{\lambda_{x}} x\right) .
$$

TABLE 2. Cases with light background wind (base is reference case in Table $1 ; u_{g}$ and $v_{g}$ are geostrophic wind along $x$ and $y$ ).

\begin{tabular}{llccc}
\hline \hline Case & Base & $u_{g}\left(\mathrm{~m} \mathrm{~s}^{-1}\right)$ & $v_{g}\left(\mathrm{~m} \mathrm{~s}^{-1}\right)$ & $t_{s}(\mathrm{~h})$ \\
\hline L4_u2 & L4 & 2 & 0 & 6 \\
L4_v2 & L4 & 0 & 2 & 6 \\
L4_u2h & L4h & 2 & 0 & 6 \\
L4_v2h & L4h & 0 & 2 & 6 \\
L8_u2 & L8 & 2 & 0 & 11 \\
L8_v2 & L8 & 0 & 2 & 11 \\
L8_u2h & L8h & 2 & 0 & 11 \\
L8_v2h & L8h & 0 & 2 & 11 \\
\hline
\end{tabular}

Large-scale atmospheric subsidence, typical of synoptic high-pressure conditions, was applied to the potential temperature profile only (Khairoutdinov and Kogan 1999), but in contrast to AS98 a much weaker, more realistic (Muschinski et al. 1999) subsidence velocity of $-2 \mathrm{~cm} \mathrm{~s}^{-1}$ was used. To compensate for the smearing effects of ABL growth, $x-z$ cross sections and vertical profiles were additionally normalized by the ABL height $z_{i}$. The Coriolis effect was disabled for simplicity.

All simulations were run with a uniform grid spacing of $50 \mathrm{~m}$, with a $9 \%$ vertical stretching above $1800 \mathrm{~m}$. The domain size was $5 \mathrm{~km}$ in the $y$ and $3.2 \mathrm{~km}$ in the $z$ directions; the width $D_{x}$ is listed along with other simulation parameters for cases with zero background wind in Table 1 and light wind in Table 2. Width $D_{x}$ was generally chosen large enough to juxtapose at least two waves within the model domain, to allow potential eddy interaction over more than one wavelength and to clearly resolve the spectral signals. Calculations were carried out until at least two or three temporal oscillation peaks were obtained. The cases, denoted with a lower case " $h$ ", are corresponding homogeneous control runs. Cases A15 and B5 were run to reproduce the results of AS98 and are labeled accordingly.

\section{Results}

TMCs have already been well investigated (e.g., AS98; Gopalakrishnan and Avissar 2000; Baidya Roy and Avissar 2000) and will therefore only be sketched here in brief. The main focus will be on the thermally induced oscillation.

Vertical profiles, $x-z$ cross sections, power spectra and time series were used for ABL flow analysis.

\section{a. Thermally induced mesoscale circulation}

Cases A15 and B5 reproduce very well the TMCs observed by AS98. For brevity, only a plot of A15 is provided to demonstrate this. Figure 1a shows $x-z$ cross sections of (i) $u$ and (ii) $w$ for case A15 $4 \mathrm{~h}, 30$ min after simulation start, which is when the simulations of AS98 ended. (Fig. 1b shall be considered later.) The pattern shows a clear low-level convergence and high-level divergence at $x=10 \mathrm{~km}$, the surface heat wave maximum, and vice versa, though 
(i)

(a)

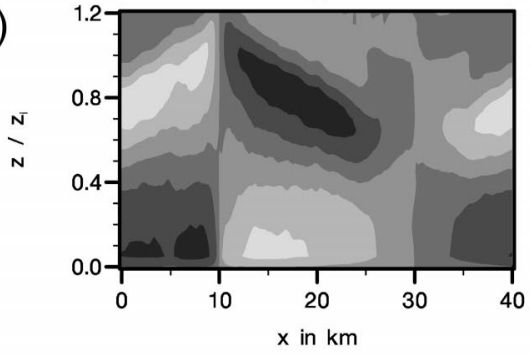

(b)

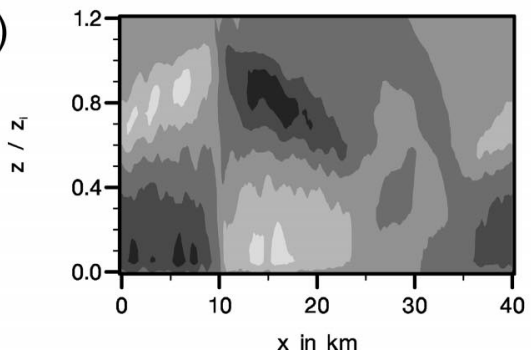

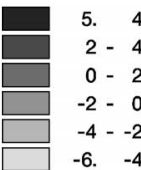

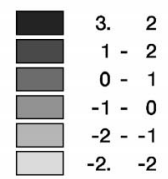

(ii)

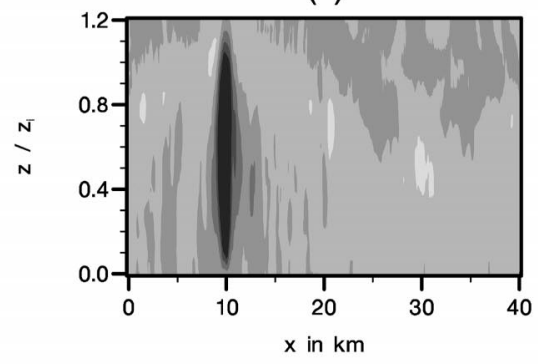

\begin{tabular}{lll}
3.8 & 1.2 \\
\hline & & 1.8
\end{tabular} $0.8-1.2$ $0.4-0.8$ $0.0-0.4$ $-0.4-0.0$ $-.6-0.4$

FIG. 1. The $x-z$ cross sections of (i) $u$ and (ii) $w\left(\mathrm{~m} \mathrm{~s}^{-1}\right)$ for case A15 (a) $4 \mathrm{~h}, 30$ min and (b) $6 \mathrm{~h}, 15$ min after simulation start, $z_{i}$ normalized, averaged in the $y$ direction and over the last $15 \mathrm{~min}$.

weaker, at $x=30 \mathrm{~km}$, the heat wave minimum. The corresponding $x-z$ cross section of $w$ exhibits a single strong updraft at $x=10 \mathrm{~km}$, only few weak adjacent updrafts at $x=0 \cdots 20 \mathrm{~km}$, and a large downdraft region at $x=20 \cdots 40 \mathrm{~km}$. Parameters $u$ and $w$ together show a single dominant open CBL circulation roll filling the entire domain, the TMC.

There are subtle differences to the results of AS98, though, but they are only small. The updraft at $x=10$ $\mathrm{km}$ (Fig. 1aii) is stronger than in AS98, which is known to be due to the double spatial resolution (Pielke 1984; Weaver et al. 2002). Consequently, the $u$ pattern in the upper portion of the boundary layer is more slanted than in AS98.

\section{b. The oscillation-Fundamentals}

\section{1) Oscillation EXISTENCE}

One of the main findings of this study is that the TMC intensity itself varies with time. AS98 already observed nonlinear vertical heat flux profiles at 4 h, 30 min (e.g., for their case A15) that clearly point at a nonquasi-steady CBL development, but they could not observe the oscillation because their simulations lasted only $4.5 \mathrm{~h}$.

In the present study, as soon as the heat wave is activated at $2 \mathrm{~h}$, the linear vertical heat flux profile of case A15 turns convex and reaches its maximum curvature at $4 \mathrm{~h}, 15 \mathrm{~min}$. Then it slowly changes to a near linear shape and turns concave with a maximum curvature at $5 \mathrm{~h}, 30 \mathrm{~min}$, returns to convex ( $7 \mathrm{~h}, 30 \mathrm{~min})$ and again concave shape ( 8 h, $45 \mathrm{~min})$. Figure 2 a shows the profiles with extreme curvature together with the corresponding linear profiles of the homogeneous control run $\mathrm{A} 15 \mathrm{~h}$.

In other words, the vertical heat flux divergence varies considerably throughout time. At times, the upper part of the CBL is heated more strongly than the lower one (convex shape), and vice versa (concave). And indeed, the vertical profiles of potential temperature in Fig. $2 b$ exhibit a sequence of stabilization followed by destabilization that corresponds well to the observed temporal variation of the heat flux profiles.

Strong evidence of the oscillation is provided by the time series of domain-averaged kinetic perturbation en$\operatorname{ergy} E^{*}$, for cases $\mathrm{A} 15$ and B5 in Fig. 3 that both describe a sine-like oscillation after the heat wave activation. Here, $E^{*}$ is defined as

$$
E^{*}=\frac{1}{n x \times n y \times n z} \sum_{i=1}^{n x} \sum_{j=1}^{n y} \sum_{k=1}^{n z} e^{*}(i, j, k),
$$

where $n x, n y$, and $n z$ are the grid dimensions and $e^{*}$ is the local kinetic perturbation energy,

$$
\begin{gathered}
e^{*}(i, j, k)=\frac{1}{2}\{[ \\
{[u(i, j, k)-\bar{u}(k)]^{2}+[v(i, j, k)-\bar{v}(k)]^{2}} \\
\left.+[w(i, j, k)-\bar{w}(k)]^{2}\right\}
\end{gathered}
$$

with $\bar{u}, \bar{v}$, and $\bar{w}(k)$ being the horizontal averages of the respective velocity components at height level $k$. The initial departure IDE* of kinetic energy $E^{*}$ of an inhomogeneous case from $E_{h}^{*}$ of the corresponding homogeneous control run is defined as

$$
\mathrm{IDE}^{*}=E^{*}\left(t_{1}\right)-E_{h}^{*}\left(t_{1}\right),
$$


(a)

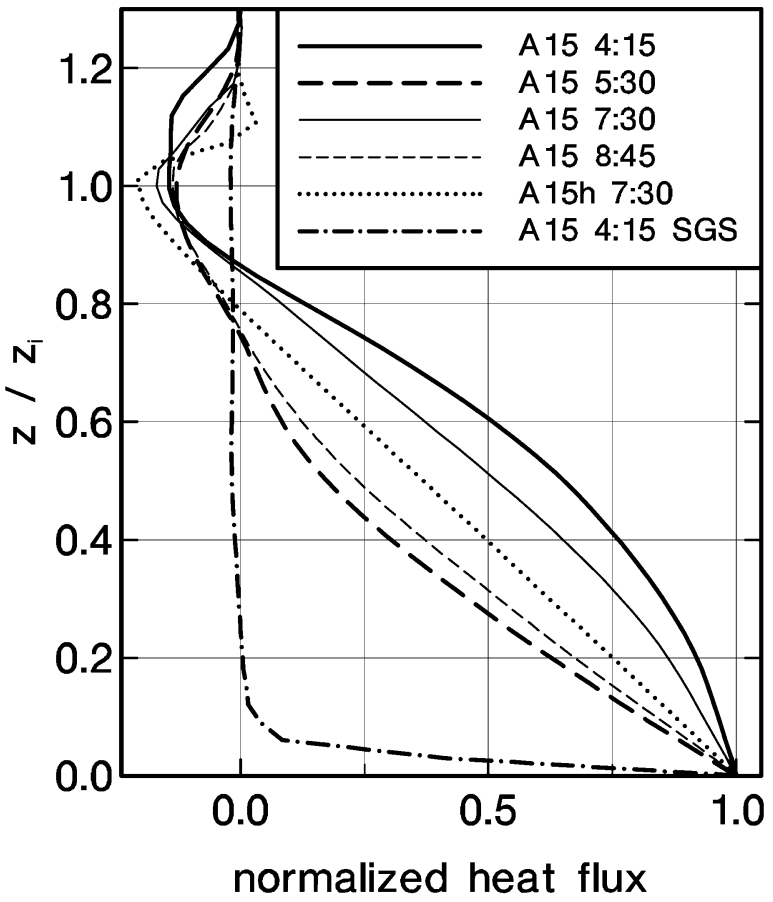

(b)

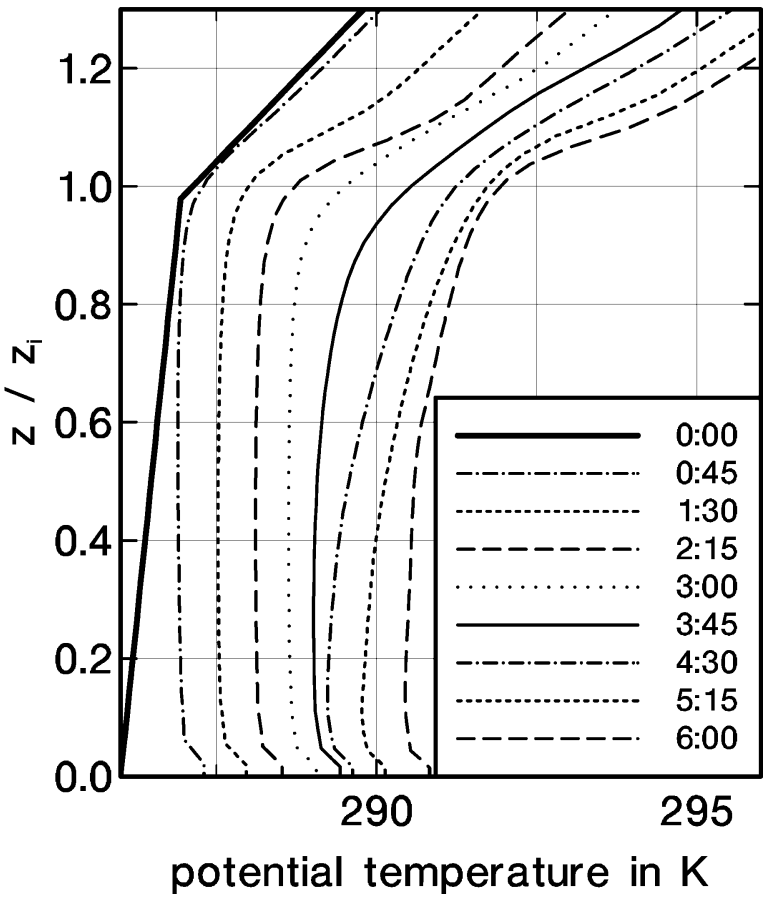

FIG. 2. Vertical profiles, $z_{i}$ normalized, of (a) normalized vertical heat flux for cases A15 and A15h and (b) potential temperature for case A15 at selected times, averaged horizontally and over the last $15 \mathrm{~min}$. The contribution of parameterized SGS heat flux (dashed-dotted lines) to the vertical heat flux is almost negligible.

where $t_{1}$ is the time when $E^{*}$ reaches its first maximum after heat wave activation (cf. example in Fig. 3).

The oscillation amplitude $A_{0}$ of $E^{*}$ in Fig. 3 decreases with time, presumably due to friction near the ground and in the entrainment layer (momentum exchange with nonmoving air aloft). (Figure 2 a shows that the maximum curvature, too, decreases with time.) Note that also the mean energy level itself is considerably increased compared with the homogeneous control runs, which is another main finding of this study and will be discussed in section $3 c(3)$. Case B5 has not only a lower forcing heat flux mean and amplitude but also exhibits smaller
$A_{0}$ than A15. It requires more time to trigger off the oscillation, and it has a longer oscillation period $T_{0}$.

The maxima of the A15 $E^{*}$ time series coincide exactly with peak TMC strength (Fig. 1a), the minima with weakest TMC flow (Fig. 1b, halved intensity).

In order to test whether the TMC oscillation depends on the chosen resolution and on the SGS model, two cases have been rerun with double resolution (25-m grid spacing; not listed in Tables 1-3). These simulations produced the same TMC oscillation (onset, phase, and amplitude; not shown) as their 50-m counterparts, which substantiates the oscillation existence.

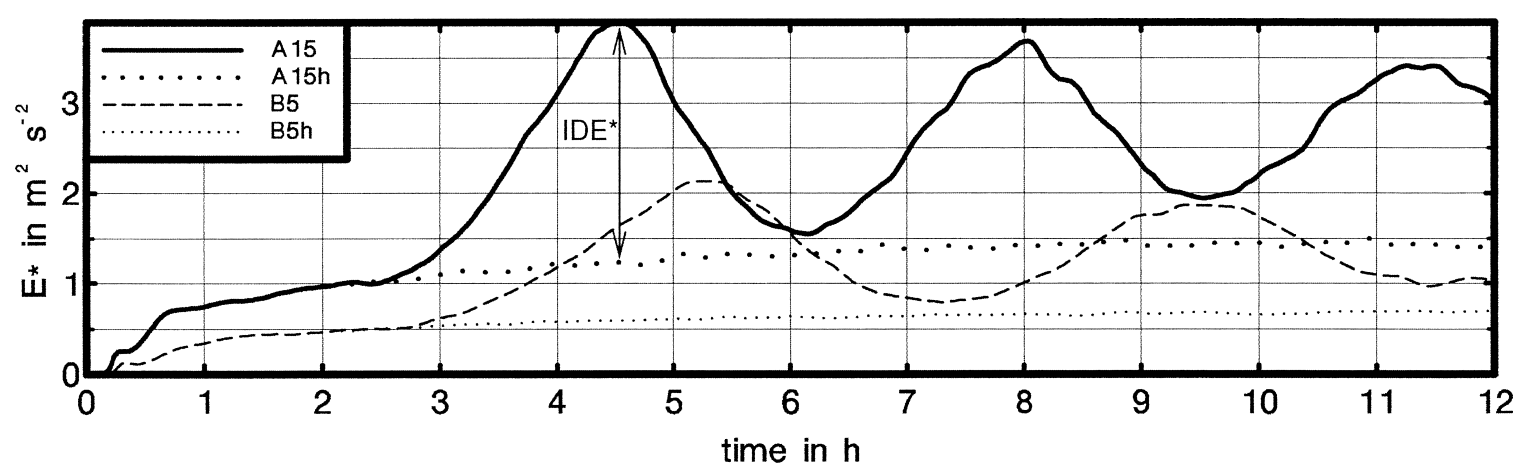

FIG. 3. Time series of kinetic perturbation energy $E^{*}$ for cases A15 and B5 and their respective homogeneous control runs. Both A15 and B5 have 40-km wavelength, but A15 has double heat flux mean and amplitude. [IDE* is an example how to derive the initial departure of kinetic energy $E^{*}$ from its corresponding homogeneous value for case A15; cf. section $3 \mathrm{~b}(1)$.] 

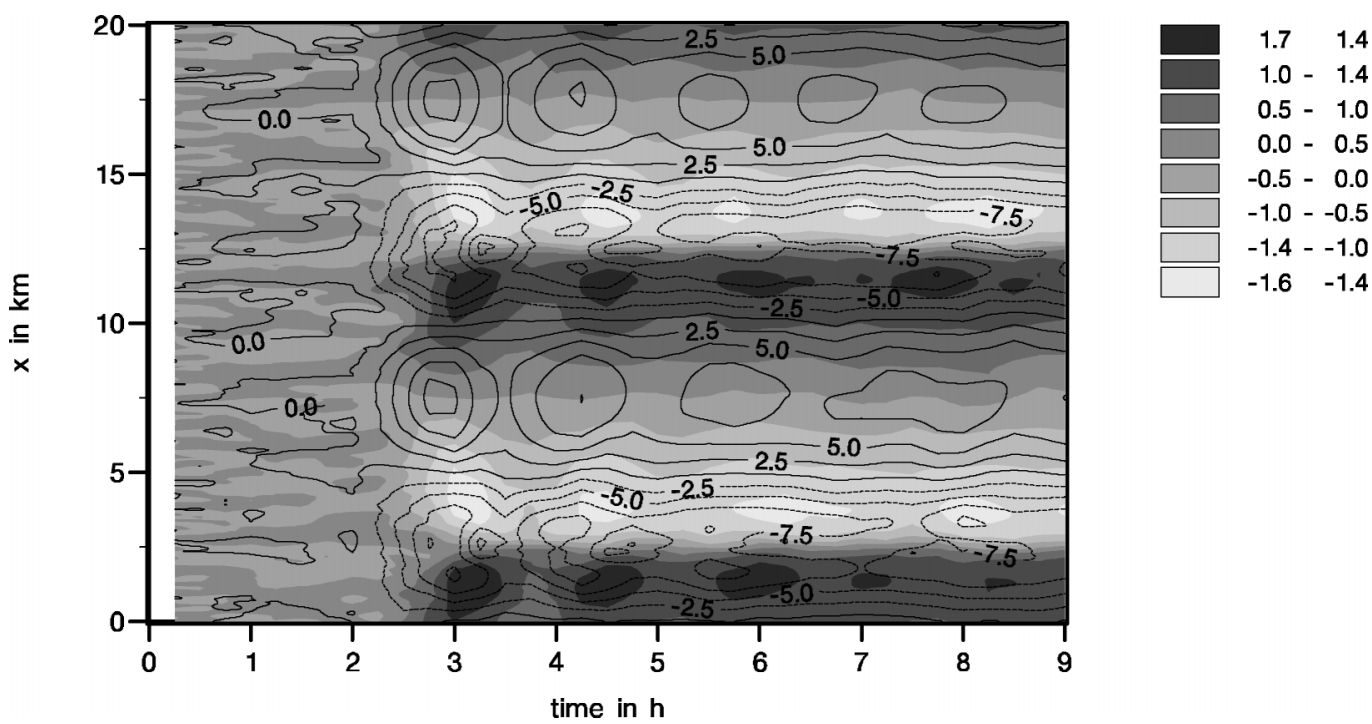

FIG. 4. 2D $x-t$ time series of perturbation pressure $p$ in $\mathrm{Pa}$ (isolines) and velocity component $u$ ( $\mathrm{m} \mathrm{s}^{-1}$; contours) for case L4. For description, see text.

\section{2) Oscillation MECHANISM-A HYPOTHESIS}

This section presents a hypothesis for the oscillation mechanism. The point is not so much the TMC onset itself (which can be attributed to horizontal surface heat flux, temperature and pressure gradients) — the key question seems to be what causes the TMC intensity to decrease (and then increase) again.

Figure 4 shows the temporal evolution of the horizontal variation of perturbation pressure $p$ and velocity component $u$ for case L4 near the ground in form of 2D $x-t$ time series of $p$ and $u$ that have been obtained in a three-step process: 1) $x-z$ cross sections of $p$ and $u\left(z_{i}\right.$ normalized, averaged in the $y$ direction and over $15 \mathrm{~min}$ ) are computed all throughout the simulation; 2) these cross sections are evaluated near the ground at height $z=0.05 z_{i}$; and 3) they are then plotted as $x$ versus $t$ time series overlay of $p$ (isolines) and $u$ (contours). Note the smoothing effect of the 15-min timeaveraged input data in step 1). When the heat wave is activated at $t=2 \mathrm{~h}$, not only the temperature gradient (not shown), but also the pressure gradient immediately starts to build up. The TMC - as represented by $u$ (and $w$, not shown)-follows with a phase lag of some 15 min. As the TMC grows in strength, the pressure gradient reaches its maximum and begins to weaken. The TMC peak strength coincides with the most rapid decrease of the pressure gradient. This cycle repeats itself in fairly regular intervals with decreasing peak strength, which indicates that $p$ and $u$ oscillate around some equilibrium state.

Figure 4 suggests that the TMC itself effectively reduces the horizontal pressure and temperature gradients that were responsible for its onset. Both gradients being reduced, the TMC itself also decreases in strength. Meanwhile, the surface heat wave of course continues, thus acting to restore the gradients. This explains why the TMC intensity then again rises to a second peak.

Note that-except for the oscillation-the pressure and velocity patterns basically remain the same until the end of the simulation and clearly reflect the forcing heat wave compared with the homogeneous pattern before $t$ $=2 \mathrm{~h}$. This points to the considerably increased mean energy level in Fig. 3.

A simple conceptual hypothesis of the TMC oscillation serves to explain the observed features: The surface heat wave causes horizontal gradients in temperature and, subsequently, perturbation pressure that induce a TMC circulation roll. However, the circulation is so strong that it levels out part of the horizontal gradients and thus effectively cuts itself off its forcing. Meanwhile, the surface heat wave continues, and when the TMC has become weak enough, the horizontal gradients start to build up again and the same cycle repeats. The amplitude of this oscillation decreases with time presumably due to friction near the ground and in the entrainment layer (momentum exchange with nonmoving air aloft).

\section{3) Oscillation OnSET}

The fact that both AS98 and the present study activated the surface heat wave instantaneously at $t=2 \mathrm{~h}$ raises the question whether the observed oscillation might not just be a numerical artifact. Indeed, the heat wave activation works like a step function in time and thus might easily trigger an artificial oscillation.

Several tests based on case L4 have been performed with different heat wave activation modes, be it full activation from the start of the simulation (L4s) or a slow linear (L4c) or sinusoidal (L4Ar) growth from zero 
TABLE 3. Cases with different heat wave activation modes, based on case L4 and plotted in Fig. 5.

\begin{tabular}{lcclc}
\hline \hline Case & $\begin{array}{c}\overline{w^{\prime} \theta_{\mathrm{av}}^{\prime}} \\
\left(\mathrm{K} \mathrm{m} \mathrm{s}^{-1}\right)\left(\mathrm{K} \mathrm{m} \mathrm{s}^{-1}\right)\end{array}$ & $\begin{array}{c}A_{x} \\
\text { Heat wave activation mode }\end{array}$ & $\begin{array}{c}t_{s} \\
(\mathrm{~h})\end{array}$ \\
\hline L4 & 0.16 & 0.15 & Instantaneously at $t=2 \mathrm{~h}$ & 9 \\
L4c & 0.16 & 0.15 & Linear from $t=1.5-2.5 \mathrm{~h}$ & 6 \\
L4s & 0.16 & 0.15 & Instantaneously from start & 6 \\
L4Ar & 0.16 & 0.10 & Sinusoidal from $t=1.5-4.5 \mathrm{~h}$ & 9 \\
L4Ab & $0.16^{*}$ & $0.10^{*}$ & *Both sinusoidal from $t=0-3 \mathrm{~h}$ & 9 \\
L4h & 0.16 & - & \multicolumn{1}{c}{-} & 11 \\
\hline
\end{tabular}

to full perturbation amplitude. One case was set to resemble the onset of the morning breeze of the land-sea breeze circulation (L4Ab). Table 3 lists the setup parameters of these tests, and Fig. 5 displays their results.

Though the oscillation amplitude and period show some variation, all cases exhibit a clear oscillation, and all finally arrive within an energy range that is significantly above the energy level of the homogeneous control run. This suggests that the activation mode itself plays only a minor role for the oscillation onset. We suggest that the horizontal gradients of temperature and pressure are crucial here: as soon as they exceed a critical threshold value, the TMC suddenly sets in and the TMC onset itself triggers off the oscillation. This compares well to the land-sea breeze circulation where the circulation also sets in suddenly and only some time after the differential heating, that is, after the horizontal gradients exceed a threshold.

Furthermore, Fig. 5 shows that the TMC onset does not necessarily depend on boundary layer turbulence being fully developed (L4s). Our results indicate that, when the perturbation wavelength $\lambda_{x}$ is sufficiently large (10 km and more), the TMC is reduced to a $2 \mathrm{D} \mathrm{me-}$ soscale phenomenon that may simply be analyzed with a 2D mesoscale model as recently demonstrated by Lee and Kimura (2001). However, analysis of the strong TMC feedback on the turbulence structure (see section 3d) still requires the use of an LES model.

Finally, Fig. 5 also indicates that there is a time scale of some $5 \sim 6 \mathrm{~h}$ required for the flow to come to equilibrium with the inhomogeneous surface heat flux forc- ing. Figures 6 and 7 (cf. the following section) show that this time scale depends on the perturbation wavelength and amplitude. In contrast to the time required for atmospheric turbulence to come to equilibrium with a change in surface forcing, which is on the order of only a few convective time scales, this time scale is much longer because it represents the time required for the mesoscale circulation to become stationary. This is not surprising because the TMC is mesoscale in both space and time.

\section{c. The oscillation-Parameter space}

In section $3 \mathrm{~b}(1)$, we showed that the oscillation $\left(A_{0}\right.$, $T_{0}$ ) varies with certain parameters. In this section we explore the parameter space and investigate how the oscillation depends on 1) perturbation wavelength $\lambda_{x}$, 2) amplitude $A_{x}$, and 3) background wind $u_{g}$ and $v_{g}$.

\section{1) IMPACT OF PERTURbation WAVELENGTH $\lambda_{x}$}

Figure 6 shows time series of $E^{*}$ for cases that differ only in $\lambda_{x}$ (and simulation time $t_{s}$ ). Their results differ in both oscillation period $T_{0}$ and amplitude $A_{0}$. The larger $\lambda_{x}$, the larger $T_{0}$ and $A_{0}$.

However, the smallest wavelengths, $\lambda_{x}=2.5$ and 5 $\mathrm{km}$ (L1, L2), do not produce clear oscillations, and their average energy level even drops below that of the homogeneous control runs (L4h, L7h).

Figure 6 further suggests that, although the initially clear oscillations of cases L3 to L8 may cease after some time, their energy level will probably still remain much higher than in the homogeneous control runs.

Figure 8 (top) shows that $T_{0}$ depends almost linearly on $\lambda_{x}$. As the oscillation amplitude $A_{0}$ is difficult to measure 1) because of its decrease with time and 2) because of the slowly rising mean energy level of the homogeneous control run; Fig. 8 (bottom) instead depicts the initial departure IDE* $^{*}$ of kinetic energy $E^{*}$ from its corresponding homogeneous value. (Figure 3 shows an example of how to derive IDE*.) However, IDE* has no clear linear relationship with $\lambda_{x}$.

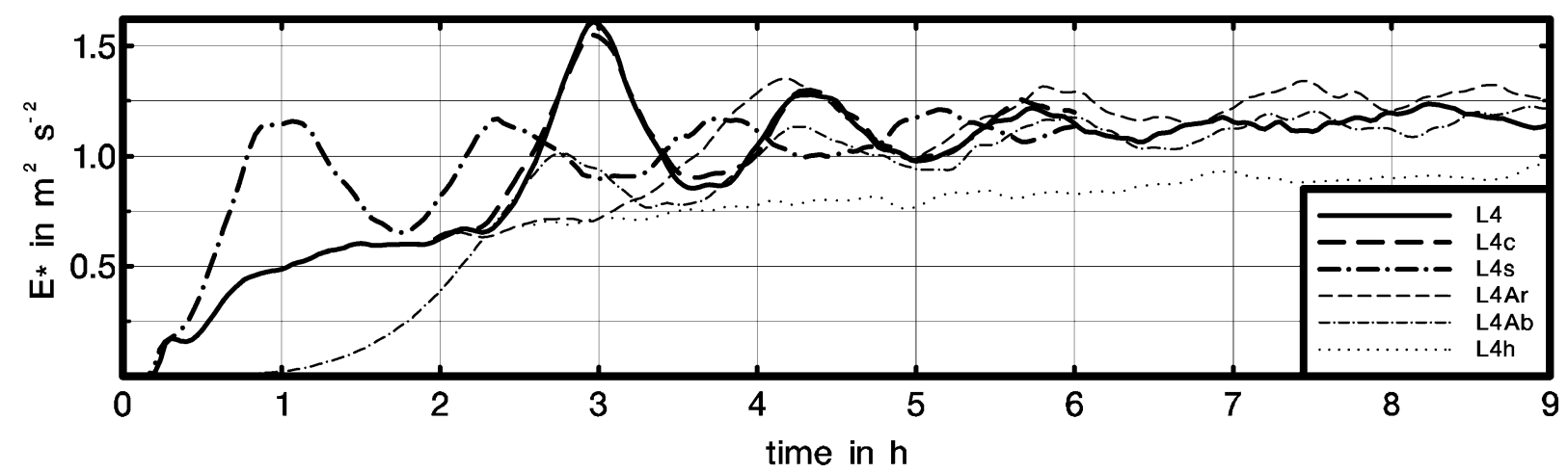

FIG. 5. Time series of kinetic perturbation energy $E^{*}$ for cases with different heat wave activation modes, based on case L4 (see Table 3 ). 


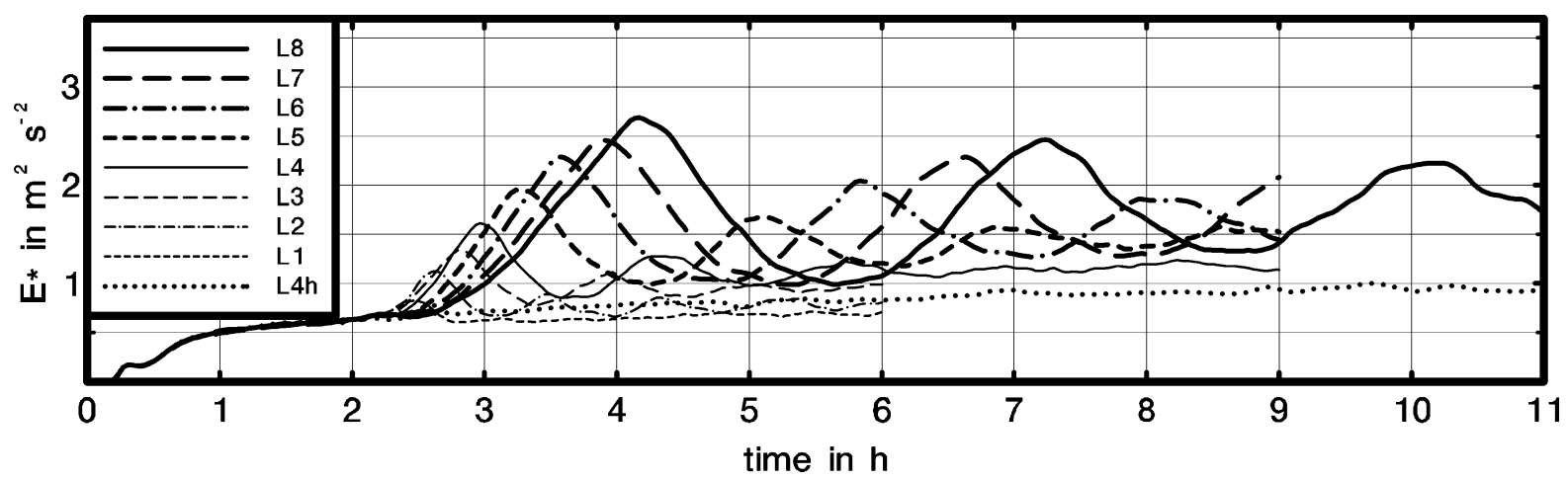

FIG. 6. Time series of kinetic perturbation energy $E^{*}$ for cases L1 to L8 with homogeneous control run L4h. Cases L1 to L8 have same heat flux mean and amplitude but differ in wavelength and simulation time.

\section{2) ImPACT OF PERTURbAtion AMPLitude $A_{x}$}

Figure 7 shows time series of $E^{*}$ for cases that differ only in $A_{x}$ (and simulation time $t_{s}$ ). Their results also differ in both $T_{0}$ and $A_{0}$. The larger $A_{x}$, the larger $A_{0}$, but the smaller $T_{0}$.

However, the smallest amplitude, $A_{x}=0.01 \mathrm{~K} \mathrm{~m} \mathrm{~s}^{-1}$ (L4D), does not produce oscillations, and its average energy level is comparable to that of the homogeneous case L4h. That is, the threshold of TMC formation [cf. $3 \mathrm{~b}(3)]$ is between $A_{x}=0.01$ and $0.03 \mathrm{~K} \mathrm{~m} \mathrm{~s}^{-1}$.

Again, the energy level of those cases with clear oscillations (L4, L4A, L4B) remains considerably higher than in the homogeneous case (L4h).

There may be a nonlinear relationship between the oscillation period $T_{0}$ and $A_{x}$ in Fig. 9 (top), but as $T_{0}$ could only be quantified for three of the five cases (the oscillation disappears in L4C and L4D), this remains speculative. IDE* (Fig. 9, bottom), however, indicates a linear dependence on $A_{x}$, though it could only be quantified for four of the five cases.

The results of $3 c(1)$ and $3 c(2)$ suggest that the oscillation period $T_{0}$ depends on the speed of the TMC onset. A large perturbation wavelength $\lambda_{x}$ (weak horizontal surface heat flux gradient, thus slow differential heating) or a small perturbation amplitude $A_{x}$ (also, slow differential heating) impede a quick TMC onset: the horizontal pressure gradients necessary for the TMC onset take longer to build up and reach the threshold.

It is worth noting that the oscillation amplitude $A_{0}$ increases not only with perturbation amplitude $A_{x}$ (as one would expect), but also with perturbation wavelength $\lambda_{x}$. This is a nonlinear effect, because the TMC forcing, the horizontal gradient decreases with wavelength. The energy cascade may serve to explain this. At wavelengths near its peak at the natural scale of convection $(2-3 \mathrm{~km})$, the horizontal gradients due to the imposed inhomogeneities compete with those generated by the largest CBL eddies in natural convection and have to overcome their inertia to set up a TMC on this scale. At large wavelengths, however, this problem may not be so severe because on these scales the natural turbulence intensity has decreased by about an order of magnitude, making TMC generation easier.

\section{3) DisCUSSION OF THE INCREASED KINETIC ENERGY LEVEL}

This section discusses possible reasons for the increased level of $E^{*}$ that can be observed in Figs. 3, 5, 6 , and 7 .

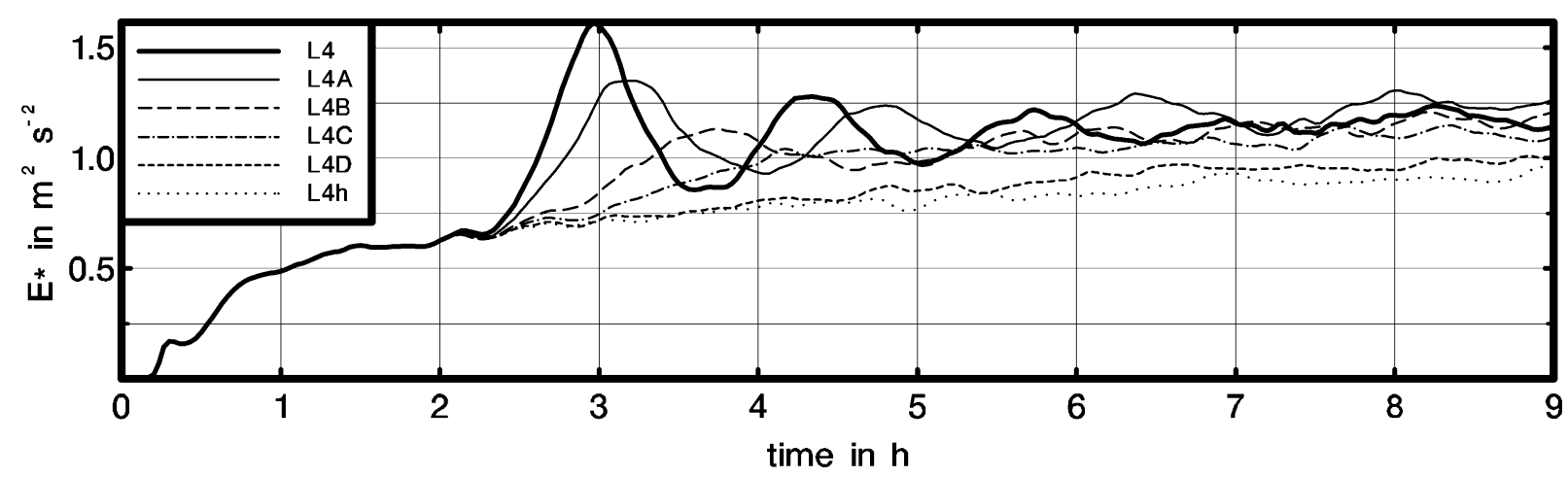

FIG. 7. Time series of kinetic perturbation energy $E^{*}$ for cases L4, L4A to L4D, and their homogeneous control run L4h. These cases differ only in heat flux amplitude. 

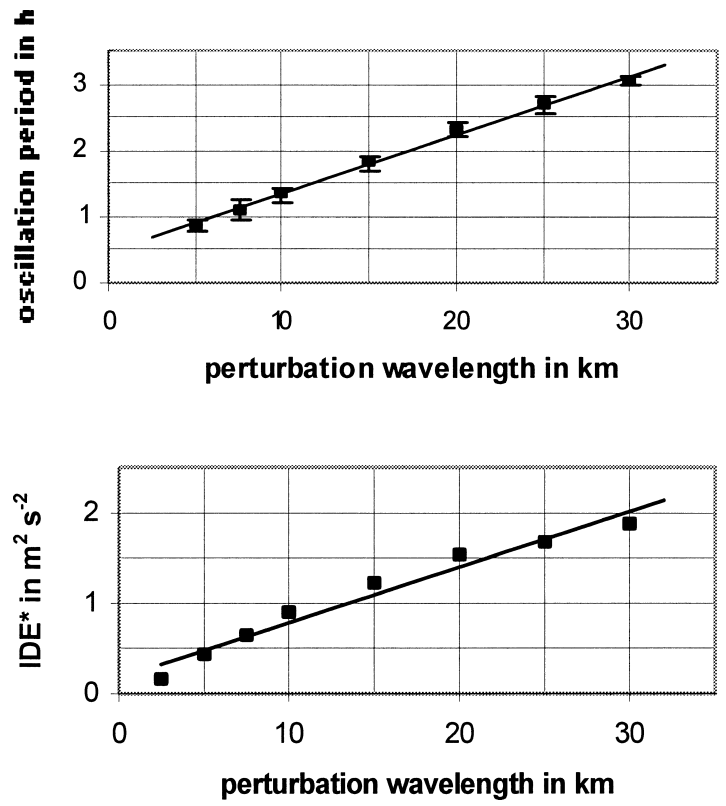

FIG. 8. Dependence of oscillation period $T_{0}$ (top) and initial departure IDE* of kinetic energy $E^{*}$ from homogeneous control run (bottom) on the perturbation wavelength $\lambda_{x}$. Linear trend lines are added. Error bars (top) indicate the variability of $T_{0}$ in the individual time series.

Souza et al. (2000) presented a theory based on the second law of thermodynamics (the surface heat flux is proportional to the vertical temperature gradient at ground level) that explains well why surface inhomogeneities enhance the CBL circulation (hence, increase the kinetic energy level). Under homogeneous conditions, the surface heat flux decreases when an air parcel advects toward an updraft because as it heats up, the difference between its own and the ground surface temperature decreases. However, in case of horizontal surface temperature gradients, the surface heat flux then decreases less rapidly. This allows higher horizontal temperature and pressure gradients.

This study directly prescribed the surface heat fluxes. Thus, while the air parcel approaches the updraft, in the inhomogeneous case the surface heat flux even increases further (as prescribed), whereas in the homogeneous case it remains constant (as prescribed). Therefore directly prescribed inhomogeneous surface heat fluxes also lead to a speed-up compared with prescribed homogeneous surface heat fluxes.

Generally, a circulation serves to balance gradients. This principle holds on all scales from the global scale down to local microscale. Convection predominantly enhances vertical flow (boundary layer mixing) because it serves to balance vertical temperature differences. Mesoscale circulations, such as the land-sea breeze circulation, predominantly enhance the horizontal flow because they serve to balance horizontal temperature and pressure gradients.

In the present study, the horizontal surface heat flux
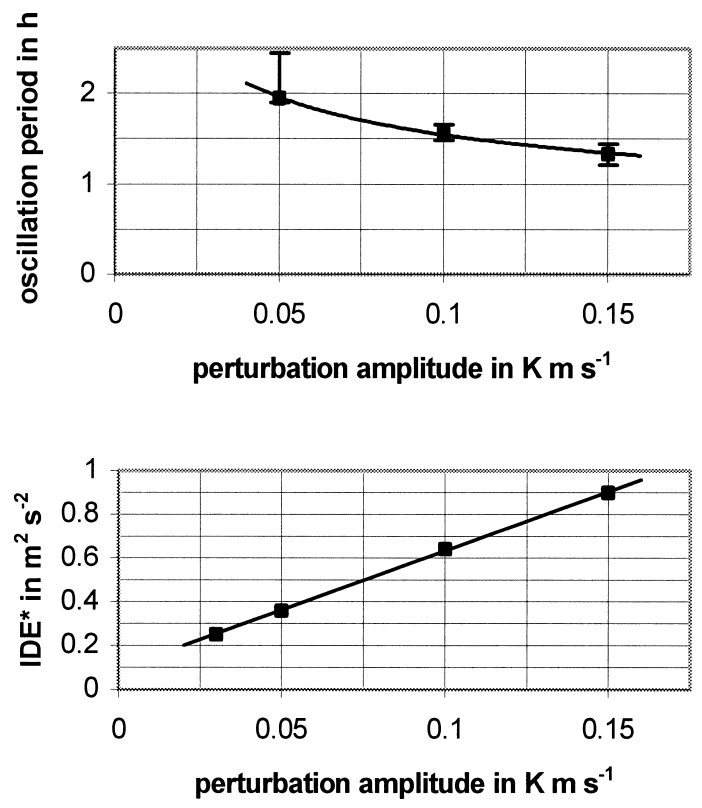

FIG. 9. Dependence of oscillation period $T_{0}$ (top) and initial departure IDE* of kinetic energy $E^{*}$ from homogeneous control run (bottom) on the perturbation amplitude $A_{x}$. A power law and a linear trend line are added, respectively. For error bars, compare with Fig. 8.

inhomogeneity differentially heats the CBL, and it thus causes a horizontal pressure gradient that gives the flow an additional degree of freedom and lets the TMC develop. This would happen even if the average horizontal surface heat flux $\overline{w^{\prime} \theta^{\prime}}$ av were zero-though, under homogeneous conditions there would then be no convection at all.

It can thus be concluded that the pronounced increase in $E^{*}$ as shown by Figs. 3, 5, 6, and 7 is inherent in the increased horizontal flow due to the TMC.

Of course the TMC increases not only the speed of horizontal flow but also the boundary layer growth, through enhanced entrainment processes. This poses the question whether enhanced entrainment may also contribute to the increase in $E^{*}$.

If there is no ambient wind, the dynamical effect of entrainment is a momentum exchange with nonmoving air in the inversion layer, and the thermal effect of entrainment is, always, a stabilization of the stratification; both effects slow down the TMC. Thus, entrainment can only intensify the TMC if there is nonzero ambient wind and if the dynamic effect of momentum exchange with the ambient wind in the inversion layer is stronger than the thermal stabilization effect. However, in the present study the boundary layer of the inhomogeneous cases grew less than $10 \%$ higher than in corresponding homogeneous control runs, which is in good agreement with earlier studies (e.g., Shen and Leclerc 1995; Raasch and Harbusch 2001). That is, entrainment processes were only slightly increased and can account for such a pronounced increase in $E^{*}$, neither for cases without ambient wind as shown by Figs. 3, 5, 6, and 7, nor for 


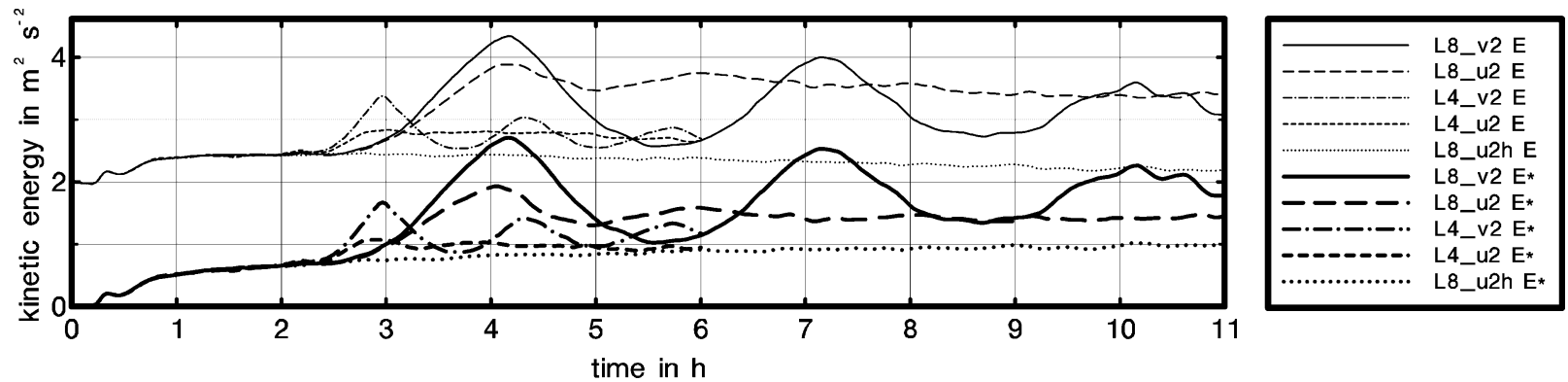

FIG. 10. Time series of total kinetic energy $E$ and perturbation energy $E^{*}$ for all cases with background wind (Table 2) and a homogeneous control run. These cases vary in wavelength, wind direction, and simulation time.

cases with ambient wind (Fig. 10) that are discussed next.

\section{4) IMPACT OF BACKGROUND WIND}

Figure 10 shows time series of kinetic energy for cases with weak background wind $\left(2 \mathrm{~m} \mathrm{~s}^{-1}\right)$ that differ in both $\lambda_{x}$ and wind direction. Because of the nonzero mean flow, total kinetic energy $\mathrm{E}$ and perturbation energy $E^{*}$ differ and are both plotted here. For simplicity, only one homogeneous control run, L8_u2h, is shown in Fig. 10, because the results of the other homogeneous control runs (L8_v2h, L4_u2h, and L4_v2h) coincide with those of L8_u2h.

In the cases with $u_{g}=2 \mathrm{~m} \mathrm{~s}^{-1}$ (L4_u2 and L8_u2; the wind blows across the surface inhomogeneity), enhanced turbulent shear in the $x$ direction smears the surface information, weakens the TMC, and rapidly dampens any initial TMC oscillations. Nevertheless E reaches significantly higher levels than in the homogeneous control run (L8_u2h).

In the cases with $v_{g}=2 \mathrm{~m} \mathrm{~s}^{-1}$ (L4_v2 and L8_v2; the wind blows along the surface inhomogeneity), the background wind has almost no effect on the TMC and the oscillation: $E^{*}$ maxima and minima of cases L4_v2 and L8_v2 (Fig. 10) are synchronous with and equal in magnitude to those of the reference cases L4 and L8 (Fig. 6). Both $E$ and $E^{*}$ of cases L4_v2 and L8_v2 reach significantly higher levels than in the homogeneous control run L8_u2h (all Fig. 10).

Questions concerning the complicated, nonlinear feedback of background wind on mesoscale circulations have received considerable interest over the past few years. Studies (e.g., by Vidale et al. 1997; Wang et al. 1998; Raasch and Harbusch 2001; Weaver and Avissar 2001; Baidya Roy and Avissar 2002; Weaver et al. 2002) indicated that the impact of background wind on TMC strength may be less significant than thought before (e.g., Wang et al. 1996; Zhong and Doran 1997, 1998). Our study adds supporting evidence to the former for TMCs triggered by 1D surface inhomogeneities under weak background winds $\left(2 \mathrm{~m} \mathrm{~s}^{-1}\right)$. TMC interaction with higher wind speeds is currently being investigated (e.g., Kim et al. 2002). Raasch and Harbusch (2001) already noted that the background wind does not necessarily weaken TMCs, only the component perpendicular to the orientation of the inhomogeneities does so. Our study supports their results but shows that even in the latter case for a weak background wind $\left(2 \mathrm{~m} \mathrm{~s}^{-1}\right)$ the kinetic energy level remains much higher than in homogeneous simulations.

\section{d. TMC spectral characteristics}

The increased average kinetic energy level described and discussed in the previous section is only the integral result of the TMC effect on CBL turbulence. This section investigates how the TMC changes the spectral properties of CBL turbulence. Does the kinetic energy rise throughout the entire spectral range, in the $x$ and $y$ directions?

Which is the general picture that one would expect of TMC power spectra?

If a power spectrum of the inhomogeneous surface heat flux were to be drawn in the $x$ direction, the heat wave would merely produce one single peak at the wavelength of the superimposed sinusoidal perturbation. However, the usual asymmetric open-cell CBL circulation-strong, narrow updrafts counterbalanced by weak, broad downdrafts-also applies to the TMC (cf. section 3a). The boundary of the asymmetric TMC cell in Fig. 1 is at $x=10 \mathrm{~km}$, the surface heat wave maximum. While the dominant scale in Fig. 1 is doubtless the forcing surface heat wavelength $\lambda_{x}$, neither $u$ nor $w$ are as symmetric as the sinusoidal forcing; that is, despite its sinusoidal forcing, the CBL does not develop a purely sinusoidal TMC. The implication for the TMC power spectra is that while a main spectral peak at $\lambda_{x}$ is, of course, to be expected, higher-order peaks are needed to account for the asymmetry.

Figure 11 shows power spectra of the velocity components $u, v$, and $w$ for case L4 and its homogeneous control run L4h at $z_{i}$-normalized heights. Cases L4 and L4h serve as representative examples for all cases. The spectra are time averaged over $t=6-9 \mathrm{~h}$, when the oscillations are fading down but the domain-averaged kinetic perturbation energy $E^{*}$ remains high (L4). The $x$ power spectra are computed in the $x$ direction and av- 
(a)

(i)

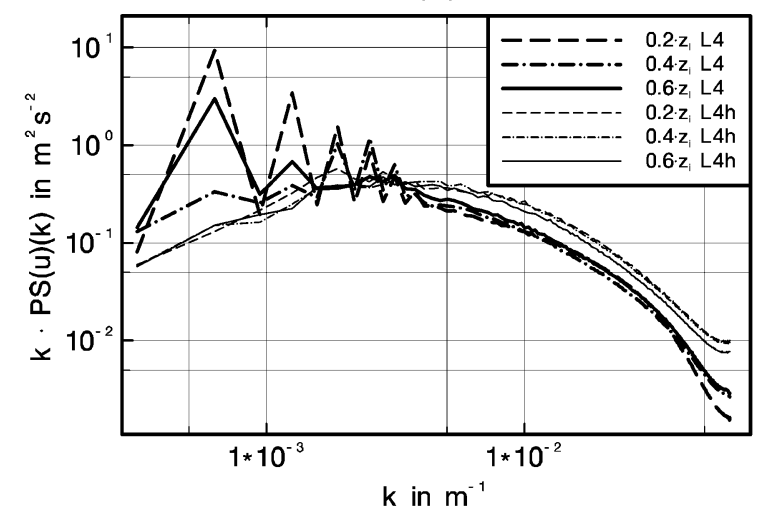

(ii)

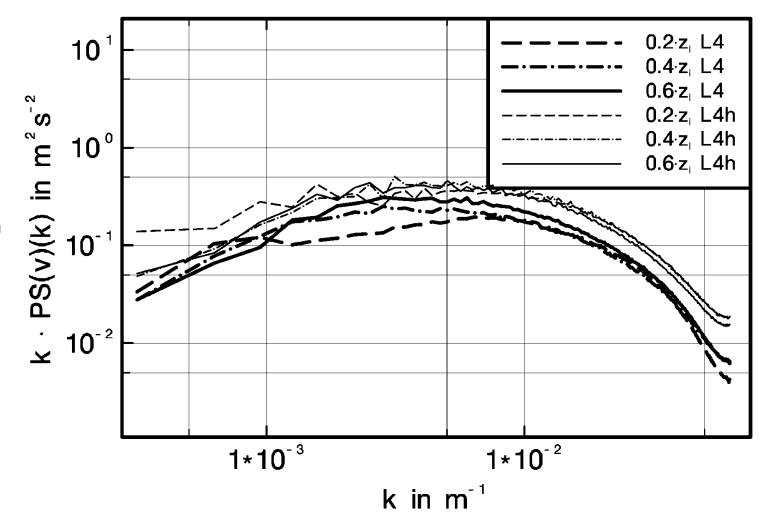

(iii)

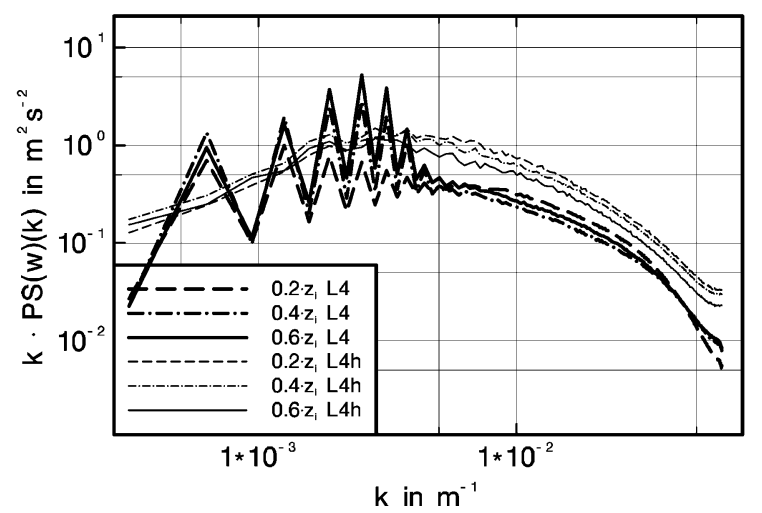

(b)
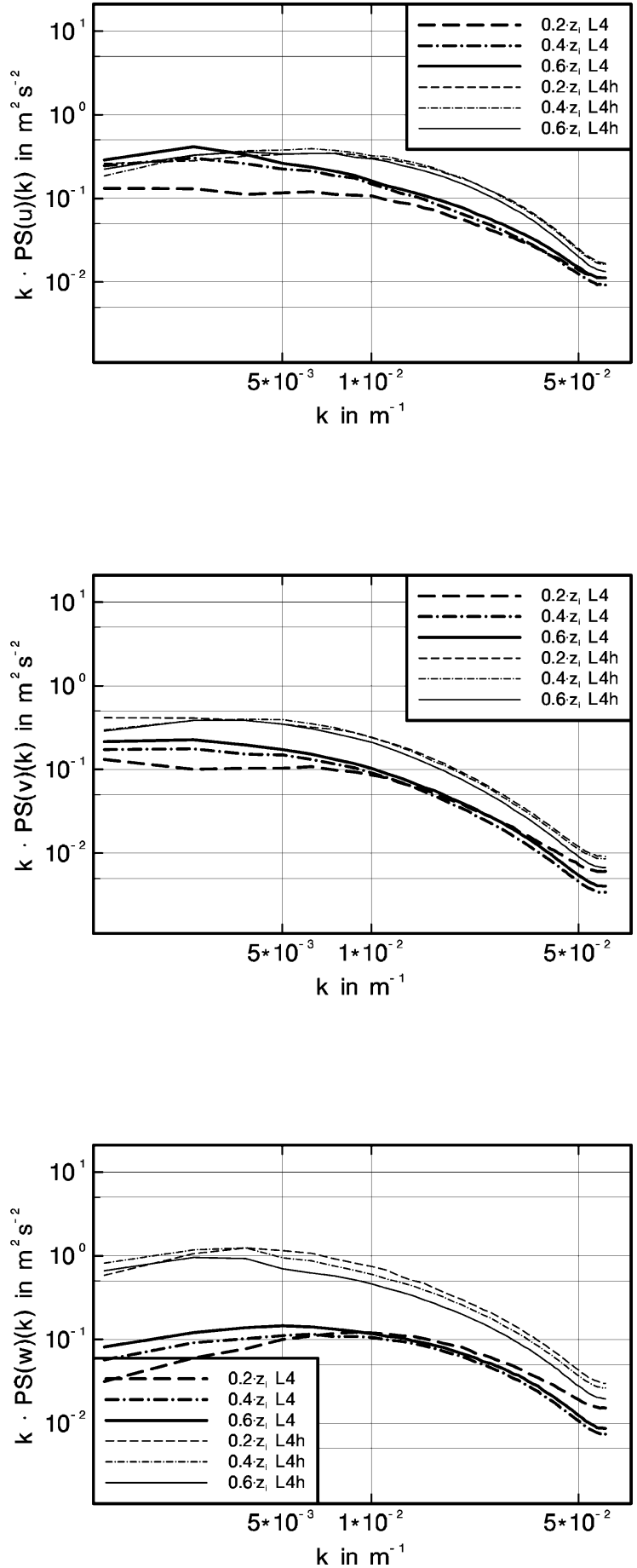

FIG. 11. Power spectra of velocities (i) $u$, (ii) $v$, and (iii) $w$, time averaged over $t=6-9$ h, calculated at $z_{i}$-normalized heights for cases L4 and L4h: (a) $x$ power spectra and (b) $y$ power spectra.

eraged along $y$ (Figs. 11ai-iii); $y$ power spectra, vice versa (Figs. 11bi-iii). Note that the spectral range differs depending on direction, because the model domain is four times wider than it is deep $(20 \mathrm{~km} \times 5 \mathrm{~km})$, which also explains why the $y$ power spectra-averaged along $x$ are much smoother than the $x$ power spectra.

The shape and magnitude of the homogeneous turbulence spectra (L4h) agree well with previous findings 
(experimental, e.g., Kaimal et al. 1976; LES, e.g., Schröter et al. 2000). The sharp drop at high wavenumbers is a typical shortcoming of LES spectra (e.g., Moeng and Wyngaard 1988). The inhomogeneous turbulence spectra (L4) differ markedly. As expected, the $x$ power spectra indeed show a main spectral peak at the wavenumber $k_{x}$ of the forcing surface heat wavelength $\lambda_{x}\left(k_{x}=2 \pi \lambda_{x}^{-1}=6.3 \times 10^{-4} \mathrm{~m}^{-1}\right)$ plus a number of harmonics, that is, spectral peaks at integral fractions of $\lambda_{x}$, which make up for the asymmetry. None of the $y$ power spectra show any sign of a TMC-related circulation in the $y$ direction, nor does the $x$ power spectrum of $v$ reveal any TMC signal. This is because the model has been set up homogeneously in the $y$ direction. Only in test runs with Coriolis effect (not listed in Tables 1-3) did a signal in the $x$ power spectra of $v$ appear after several hours.

Probably the most interesting information of Fig. 11 is that the spectral energy does not rise as a whole, as the time series of domain-averaged kinetic perturbation energy $E^{*}$ might have suggested. The direct comparison of the inhomogeneous (L4) and the homogeneous (L4h) power spectra shows that 1) the spectral energy increases in the $x$ but decreases in the $y$ direction, and 2) the TMC changes the turbulence intensity in a variable selective, direction selective, and scale selective way. The panels also reveal subtle differences between the different variables and between the spectra directions.

To start with the fairly obvious, $y$ power spectra of $u, v$, and $w$ (Figs. 11bi-iii) show that turbulence in the $y$ direction is suppressed considerably throughout the entire spectral range for all variables, only $u$ at small wavenumbers $\left(k<5 \times 10^{-3} \mathrm{~m}^{-1}\right)$ forms a possible exception. On the logarithmic scale the difference is not so striking, but turbulence is generally suppressed by a factor of about $2-5, w$ is even suppressed by up to an order of magnitude. Furthermore, $x$ power spectra of $u$, $v$, and $w$ at large wavenumbers $\left(k>5 \times 10^{-3} \mathrm{~m}^{-1}\right)$ give a similar picture: on the small scales, turbulence is suppressed even in the $x$ direction, though only by a factor of about 2 (Figs. 11ai-iii).

The TMC's large-scale subsidence over most of the model domain damps convection equally in all directions, which is why the power spectra almost unanimously show suppressed turbulence intensity. The only exception is formed by the large scales of $u$ and $w$, where the TMC completely changes the CBL turbulence structure. The dominant mesoscale circulation shows up as markedly increased turbulence intensity in the $x$ power spectra of $u$ and $w$, but there are three notable differences between $u$ and $w$.

First, $u$ has either increased intensity (harmonics at the spectral peaks) or normal intensity (nonharmonics between the spectral peaks), but $w$ has either increased intensity (harmonics at the spectral peaks) or decreased intensity (nonharmonics between the spectral peaks) compared with the homogeneous control run. In other words, the CBL's usual random updrafts are weaker and far less randomly distributed in the presence of a TMC (L4) than under homogeneous conditions (L4h): updrafts are almost fixed to the surface heat wave maxima where they are stronger than usual; elsewhere the updrafts are weaker than normal (cf. Fig. 1).

Second, the harmonics of $u$ are much weaker than those of $w$. This is because $u$ is less asymmetric then $w$. The $u$ pattern still matches the symmetric sinusoidal forcing fairly well, both in terms of horizontal extent $(5 \mathrm{~km})$ and equal magnitude of positive and negative values of $u$. The $w$ pattern, however, does not: updrafts, though located at the surface heat wave maxima, are very narrow $(1.5 \mathrm{~km})$ and strong opposed to broad $(8.5$ $\mathrm{km}$ ) and weak downdrafts. This asymmetry of different horizontal extent, as well as different magnitude of positive and negative values of $w$ requires far more and stronger harmonics for a spectral decomposition. Here, it would be misleading to say that the harmonics imply that thermals exist on their respective scales; the harmonics are but a sign that this $w$ pattern does not really suit well for a Fourier analysis.

Third, while $w$ peaks are distinct at all height levels, $u$ peaks are weak at 0.6 and especially at $0.4 z_{i}$. This is because $u$ has its transition between low-level convergence and high-level divergence at the height interval from about $0.4-0.6 z_{i}$, where the horizontal structure is less pronounced (comparable with case A15; cf. Fig. 1 ), whereas the $w$ pattern naturally does not have such height dependence.

\section{Summary and conclusions}

Oscillations have been identified that set in when TMCs on scales of $5 \mathrm{~km}$ and more develop. Their period and amplitude depend both on the scale and the intensity of the thermal surface forcing. With the TMC onset, the kinetic energy level increases markedly and remains significantly higher than under homogeneous conditions. Large-scale subsidence associated with the TMC damps convection equally in all directions.

These new findings might call into question turbulence parameterizations employed by GCMs and other larger-scale models even if they rely on homogeneous control runs of high-resolution models. The size of a typical grid box is on the order of the total domain size of the present study. Here, due to the periodic inhomogeneous forcing, the domain-averaged surface heat flux value exactly equals that of a completely homogeneous model run-but the kinetic energy, for instance, on which many turbulence parameterizations are based, deviates considerably. This affects the turbulent transport processes and needs to be accounted for in largerscale models.

The highly idealized character of this study and its predecessors (AS98; Gopalakrishnan and Avissar 2000; Baidya Roy and Avissar 2000) calls for further research using more realistic surface conditions, for example diurnal heat flux variations, a superposition of inhomo- 
geneities of different wavelength, amplitude, dimension and form, and the like. Such work is currently under way within the German framework programs Deutsches Klimaforschungsprogramm (DEKLIM) and Atmosphärenforschung 2000 (AFO 2000). The influence of higher wind speeds on the TMC is currently being addressed by Kim et al. (2002) and the influence of soil moisture by Patton et al. (2002).

Experimental proof of TMCs and oscillations is likely to be impeded by the high degree of complexity of natural landscapes and diurnal heat flux variations. Nevertheless, we would expect an atmospheric response on the scale of strongest surface inhomogeneity. TMCs may occur, for example, over Antarctic coastal polynyas (Kottmeier and Engelbart 1992), where large ice water patches might allow TMCs and oscillations even to persist over some days during the polar winter. TMC-like features that may in part be due to differential surface heating have already been observed along large rivers (Oliveira and Fitzjarrald 1993; Fitzjarrald et al. 2002), over continental lakes (Yoshino 1975, chapter 3.4; Segal et al. 1997) or over patches of different land use under light daytime winds (Angevine et al. 2003; Banta and White, 2003), which suggests that under favorable atmospheric conditions (e.g., light ambient wind), TMCs can occur rather frequently and their effects are worth considering for parameterization in larger-scale atmospheric models.

Acknowledgments. This research was supported by the German National Merit Foundation (Studienstiftung des deutschen Volkes) in Bonn, Germany, and German BMBF Grants 07ATF37-UH and 01LD0103. Calculations were performed on the SGI/CRAY-T3Es of the Konrad-Zuse-Zentrum für Informationstechnik (ZIB) in Berlin, Germany, and the Regionales Rechenzentrum für Niedersachsen (RRZN) in Hannover, Germany. The authors wish to thank M. Kanda and R. Moriwaki (Tokyo Institute of Technology, Tokyo, Japan); Y. Noh (Yonsei University, Seoul, Korea); and D. Etling, T. Hauf, M. Herold, and J. Uhlenbrock (University of Hannover, Hannover, Germany) for their valuable comments and suggestions. We are also grateful for the comments of Roni Avissar and two anonymous reviewers.

\section{REFERENCES}

Albertson, J. D., W. P. Kustas, and T. M. Scanlon, 2001: Large-eddy simulation over heterogeneous terrain with remotely sensed surface conditions. Water Resour. Res., 37, 1939-1953.

Angevine, W. M., A. B. White, C. J. Senff, M. Trainer, R. M. Banta, and M. A. Ayoub, 2003: Urban-rural contrasts in mixing height and cloudiness over Nashville in 1999. J. Geophys. Res., 108, 4092, doi:10.1029/2001JD001061.

Arnfield, A. J., 2000: Micro- and mesoclimatology. Prog. Phys. Geogr., 24, 261-271.

Arola, A., 1999: Parameterization of turbulent and mesoscale fluxes for heterogeneous surfaces. J. Atmos. Sci., 56, 584-598.

Atkinson, B. W., 1981: Meso-Scale Atmospheric Circulations. Academic Press, $495 \mathrm{pp}$.
Avissar, R., 1998: Which type of soil-vegetation-atmosphere transfer scheme is needed for general circulation models: A proposal for a higher-order scheme. J. Hydrol., 212-213, 136-154.

—_, and R. A. Pielke, 1989: A parameterization of heterogeneous land surfaces for atmospheric numerical models and its impact on regional meteorology. Mon. Wea. Rev., 117, 2113-2136.

_- and F. Chen, 1993: Development and analysis of prognostic equations for mesoscale kinetic energy and mesoscale (subgrid scale) fluxes for large-scale atmospheric models. J. Atmos. Sci., 50, 3751-3774.

_ ground-surface heat flux patchiness affects the convective boundary layer using large-eddy simulation. J. Atmos. Sci., 55, 2666-2689.

Baidya Roy, S., and R. Avissar, 2000: Scales of response of the convective boundary layer to land-surface heterogeneity. Geophys. Res. Lett., 27, 533-536.

_ and - 2002: Impact of land use/land cover change on regional hydrometeorology in Amazonia. J. Geophys. Res., 107, 8037, doi:10.1029/2000JD000266.

Banta, R. M., and A. B. White, 2003: Mixing-height differences between land-use types: Dependence on wind speed. J. Geophys. Res., 108, 4321, doi:10.1029/2002JD002748.

Belcher, S. E., and J. C. R. Hunt, 1998: Turbulent flow over hills and waves. Annu. Rev. Fluid Mech., 30, 507-538.

Cai, X.-M., 1999: Large-eddy simulation of the convective boundary layer over an idealized patchy urban surface. Quart. J. Roy. Meteor. Soc., 125, 1427-1444.

Dalu, G. A., and R. A. Pielke, 1993: Vertical fluxes generated by mesoscale atmospheric flow induced by thermal inhomogeneities in the PBL. J. Atmos. Sci., 50, 919-926.

_ - — P. L. Vidale, and M. Baldi, 2000: Heat transport and weakening of atmospheric stability induced by mesoscale flows. J. Geophys. Res., 105 (D7), 9349-9363.

Deardorff, J. W., 1980: Stratocumulus-topped mixed layers derived from a three-dimensional model. Bound.-Layer Meteor., 18, 495-527.

Fitzjarrald, D. R., O. L. L. Moraes, R. K. Sakai, R. M. Staebler, O. C. Acevedo, M. Czikowsky, and R. da Silva, 2002: Landscape heterogeneity and regional climatic variability in the east central Amazon region. Extended Abstracts, 15th Symp. on Boundary Layers and Turbulence, Wageningen, Netherlands, Amer. Meteor. Soc., 660-661.

Giorgi, F., and R. Avissar, 1997: Representation of heterogeneity effects in Earth system modeling: Experience from land surface modeling. Rev. Geophys., 35, 413-438.

Gopalakrishnan, S. G., and R. Avissar, 2000: An LES study of the impacts of land surface heterogeneity on dispersion in the convective boundary layer. J. Atmos. Sci., 57, 352-371.

— S. Baidya Roy, and R. Avissar, 2000: An evaluation of the scale at which topographical features affect the convective boundary layer using large eddy simulations. J. Atmos. Sci., 57, 334-351.

Hadfield, M. G., W. R. Cotton, and R. A. Pielke, 1991: Large-eddy simulation of thermally forced circulations in the convective boundary layer. Part I: A small scale circulation with zero wind. Bound.-Layer Meteor., 57, 79-114.

$\ldots, \ldots$, and ——, 1992: Large-eddy simulation of thermally forced circulations in the convective boundary layer. Part II: The effect of changes in wavelength and wind speed. Bound.-Layer Meteor., 58, 307-327.

Hechtel, L. M., C.-H. Moeng, and R. B. Stull, 1990: The effects of nonhomogeneous surface fluxes on the convective boundary layer: A case study using large-eddy simulation. J. Atmos. Sci., 47, $1721-1741$.

Kaimal, J. C., J. C. Wyngaard, D. A. Haugen, O. R. Coté, Y. Izumi, S. J. Caughey, and C. J. Readings, 1976: Turbulence structure in the convective boundary layer. J. Atmos. Sci., 33, 2152-2169.

Khairoutdinov, M. F., and Y. L. Kogan, 1999: A large eddy simulation model with explicit microphysics: Validation against aircraft ob- 
servations of a stratocumulus-topped boundary layer. J. Atmos. Sci., 56, 2115-2131.

Kim, H.-J., Y. Noh, and S. Raasch, 2002: Interaction between wind and temperature fields under the heterogeneous heat flux in the planetary boundary layer. Extended Abstracts, 15th Symp. on Boundary Layers and Turbulence, Wageningen, Netherlands, Amer. Meteor. Soc., 614-615.

Kottmeier, C., and D. Engelbart, 1992: Generation and atmospheric heat exchange of coastal polynyas in the Weddell Sea. Bound. Layer Meteor., 60, 207-234.

Lee, S.-H., and F. Kimura, 2001: Comparative studies in the local circulations induced by land-use and by topography. Bound.Layer Meteor., 101, 157-182.

Liu, Y., C. P. Weaver, and R. Avissar, 1999: Toward a parameterization of mesoscale fluxes and moist convection induced by landscape heterogeneity. J. Geophys. Res., 104 (D16), 19 515-19 533.

Lynn, B. H., and W.-K. Tao, 2001: A parameterization for the triggering of landscape-generated moist convection. Part II: Zeroorder and first-order closure. J. Atmos. Sci., 58, 593-607.

_ - F. Abramopoulos, and R. Avissar, 1995: Using similarity theory to parameterize mesoscale heat fluxes generated by subgrid-scale landscape discontinuities in GCMs. J. Climate, 8, 932-951.

Mahrt, L., 2000: Surface heterogeneity and vertical structure of the boundary layer. Bound.-Layer Meteor., 96, 33-62.

_ J. Jun, D. Vickers, J. I. MacPherson, J. R. Pederson, and R. L. Desjardins, 1994: Observation of fluxes and inland breezes over a heterogeneous surface. J. Atmos. Sci., 51, 2165-2178.

Moeng, C.-H., and J. C. Wyngaard, 1988: Spectral analysis of largeeddy simulations of the convective boundary layer. J. Atmos. Sci., 45, 3573-3587.

Muschinski, A., P. B. Chilson, S. Kern, J. Nielinger, G. Schmidt, and T. Prenosil, 1999: First frequency-domain interferometry observations of large-scale vertical motion in the atmosphere. $J$. Atmos. Sci., 56, 1248-1258.

Oliveira, A. P., and D. R. Fitzjarrald, 1993: The Amazon river breeze and the local boundary layer. I: Observations. Bound.-Layer Meteor., 63, 141-162.

Patton, E. G., P. P. Sullivan, and C.-H. Moeng, 2002: The influence of large-scale soil moisture heterogeneity on wetting and drying planetary boundary layers. Extended Abstracts, 15th Symp. on Boundary Layers and Turbulence, Wageningen, Netherlands, Amer. Meteor. Soc., 614-615.

Pielke, R. A., 1984: Mesoscale Meteorological Modeling. Academic Press, $612 \mathrm{pp}$.

_ 2001: Influence of the spatial distribution of vegetation and soils on the prediction of cumulus convective rainfall. Rev. Geophys., 39, 151-177.

— G. A. Dalu, J. S. Snook, T. J. Lee, and T. G. F. Kittel, 1991: Nonlinear influence of mesoscale land use on weather and climate. J. Climate, 4, 1053-1069.

Raasch, S., cited 2002: PALM group. [Available online at http:// www.muk.uni-hannover.de/ raasch/PALM_group/.]

—_ and D. Etling, 1998: Modeling deep oceanic convection: Largeeddy simulation in comparison with laboratory experiments. $J$. Phys. Oceanogr., 28, 1786-1802.

— and their effects caused by small-scale surface inhomogeneities using large-eddy simulations. Bound.-Layer Meteor., 101, 3159.

_ , and M. Schröter, 2001: PALM-A large-eddy simulation model performing on massively parallel computers. Meteor. Z., 10, 363-372.

Schröter, M., J. Bange, and S. Raasch, 2000: Simulated airborne flux measurements in a LES generated convective boundary layer. Bound.-Layer Meteor., 95, 437-456.

Segal, M., and R. W. Arritt, 1992: Nonclassical mesoscale circulations caused by surface sensible heat-flux gradients. Bull. Amer. Meteor. Soc., 73, 1593-1604.

—- M. Leuthold, R. W. Arritt, C. Anderson, and J. Shen, 1997: Small lake daytime breezes: Some observational and conceptual evaluations. Bull. Amer. Meteor. Soc., 78, 1135-1147.

Shaw, W. J., and J. C. Doran, 2001: Observations of systematic boundary layer divergence patterns and their relationship to land use and topography. J. Climate, 14, 1753-1764.

Shen, S., and M. Leclerc, 1995: How large must surface inhomogeneities be before they influence the convective boundary layer structure? A case study. Quart. J. Roy. Meteor. Soc., 121, 12091228.

Souza, E. P., N. O. Renno, and M. A. Silva Dias, 2000: Convective circulations induced by surface heterogeneities. J. Atmos. Sci., 57, 2915-2922.

Vidale, P. L., R. A. Pielke, L. T. Steyaert, and A. Barr, 1997: Case study modeling of turbulent and mesoscale fluxes over the BOREAS region. J. Geophys. Res., 102 (D24), 29 167-29 188.

Wang, J., R. L. Bras, and E. A. B. Eltahir, 1996: A stochastic linear theory of mesoscale circulation induced by the thermal heterogeneity of the land surface. J. Atmos. Sci., 53, 3349-3366.

_ - E. A. B. Eltahir, and R. L. Bras, 1998: Numerical simulations of nonlinear mesoscale circulations induced by the thermal heterogeneities of land surface. J. Atmos. Sci., 55, 447-464.

Weaver, C. P., and R. Avissar, 2001: Atmospheric disturbances caused by human modification of the landscape. Bull. Amer. Meteor. Soc., 82, 269-281.

__ S. Baidya Roy, and R. Avissar, 2002: Sensitivity of simulated mesoscale atmospheric circulations resulting from landscape heterogeneity to aspects of model configuration. J. Geophys. Res., 107, 8041, doi:10.1029/2001JD000376.

Wood, N., 2000: Wind flow over complex terrain: A historical perspective and the prospect for large-eddy modelling. Bound.-Layer Meteor., 96, 11-32.

Yoshino, M. M., 1975: Climate in a Small Area. University of Tokyo Press, $549 \mathrm{pp}$

Zeng, X., and R. A. Pielke, 1995: Landscape-induced atmospheric flow and its parameterization in large-scale numerical models. J. Climate, 8, 1156-1177.

Zhong, S., and J. C. Doran, 1997: A study of the effects of spatially varying fluxes on cloud formation and boundary layer properties using data from the Southern Great Plains cloud and radiation testbed. J. Climate, 10, 327-341.

- and _ 1998: An evaluation of the importance of surface flux variability on GCM-scale boundary layer characteristics using realistic meteorological and surface forcing. J. Climate, 11, $2774-2788$ 